\title{
Microbial mediated preparation, characterization and optimization of gold nanoparticles
}

\author{
Hamed Barabadi ${ }^{1}$, Soheila Honary ${ }^{1 *}$, Pouneh Ebrahimi ${ }^{2}$, Milad Ali Mohammadi ${ }^{1}$, Ahad \\ Alizadeh $^{3}$, Farzaneh Naghibi ${ }^{4}$ \\ ${ }^{1}$ Mazandaran University of Medical Sciences, School of pharmacy, Sari, Iran. \\ ${ }^{2}$ Department of Chemistry, School of Basic Sciences, Golestan University, Gorgan, Iran. \\ ${ }^{3}$ Department of Epidemiology and Biostatistics, School of Health, \\ Tehran University of Medical Sciences, Tehran, Iran. \\ ${ }^{4}$ School of Traditional Medicine, Shaheed Beheshti University of Medical Sciences, Tehran, Iran.
}

Submitted: August 13, 2013; Approved: April 17, 2014.

\begin{abstract}
The need for eco-friendly and cost effective methods for nanoparticles synthesis is developing interest in biological approaches which are free from the use of toxic chemicals as byproducts. This study aimed to biosynthesize and optimize the size of gold nanoparticles which produced by biotechnological method using Penicillium crustosum isolated from soil. Initially, Penicillium crustosum was grown in fluid czapek dox broth on shaker at $28^{\circ} \mathrm{C}$ and $200 \mathrm{rpm}$ for ten days and then the supernatant was separated from the mycelia to convert $\mathrm{AuCl}_{4}$ solution into gold nanoparticles. The synthesized nanoparticles in the optimum conditions were formed with fairly well-defined dimensions and good monodispersity. The characterizations were done by using different methods (UV-Visible Spectroscopy, Fluorescence, FT-IR, AFM (Atomic Force Microscopy) and DLS (Dynamic Light Scattering). The bioconversion was optimized by Box-Behnken experimental design. The results show that the effective factors in this process were concentration of $\mathrm{AuCl}_{4}, \mathrm{pH}$ of medium and temperature of shaker incubator. The $\mathrm{R}^{2}$ value was calculated to be 0.9999 indicating the accuracy and ability of the polynomial model. It can be concluded that the use of multivariate analysis facilitated to find out the optimum conditions for the biosynthesis of gold nanoparticles induced by Penicillium crustosum in a time and cost effective process. The current approach suggested that rapid synthesis of gold nanoparticles would be suitable for developing a biological process for mass scale production of formulations.
\end{abstract}

Key words: biosynthesis, optimization, gold nanoparticles, box-behnken design, Penicillium crustosum.

\section{Introduction}

Nanotechnology has attracted a great interest in development of metallic nanoparticles by various physical and chemical processes. But there is an urgent need to find an alternative method due to the unsatisfying conditions of these methods (e.g. high amount of toxic chemicals and high temperature) (Priyadarshini et al., 2013). Green chemistry approach emphasizes the usage of natural microorganisms and offers a cheaper, lighter, reliable, nontoxic and eco-friendly process (Honary et al., 2013). Different microorganisms ranging from bacteria to fungi and even plants could produce nano-scaled metals in intracellular and/or extracellular process (Mohanpuria et al., 2008). Fungi might have significantly higher productivity of nanoparticles in biosynthetic approaches owing to their much higher amounts of proteins secretion (Ahmad et al., 2003) which could prove to be a potential source for the extracellular synthesis of different nanoparticles without using toxic chemicals. These advantages made fungi more suit- 
able for large-scale production (Ahmad et al., 2003; Sagar and Ashok, 2012). In addition, the extracellular biosynthesis, using fungi, could also make downstream processing much easier than bacteria (Sagar and Ashok, 2012). However, the enzyme nitrate reductase is found to be responsible for the synthesis of nanoparticles in fungi (Honary et al., 2012a, 2013a), The extracellular production of metallic nanoparticles is still continually emerging in order to understand the mechanisms of synthesis, easy downstream processing and biocompatible processing (Zhang et al., 2011). Extracellular biosynthesis of silver nanoparticles by Aspergillus niger isolated from soil has been reported by Gade et al. (2008). Recent evidence suggests that A. niger strains do produce potent mycotoxins called ochratoxin A (Schuster et al., 2002). Biological synthesis of silver nanoparticles using Aspergillus jlavus was studied by Vigneshwaran et al. (2007). Aspergillus flavus is the second most common agent of aspergillosis, the first being A. fumigatus. A. flavus may invade arteries of the lung or brain and cause infarction. Neutropenia predisposes to Aspergillus infection. It also produces a toxin (aflatoxin) which is one of the aetiological agents for hepatocellular carcinoma (Rai and Kovic 2010). Fusarium solani isolated from infected onion was used for the biosynthesis of silver nanoparticles (Ingle et al., 2009). F. solani may also cause endocarditis and lung disease, and has been shown to be an allergen (Seifert 1996). According to the above facts, it is more eco-friendly to work with a medical-safer genus of fungi. Penicillium is a mold that is widely distributed in nature, and is often found living on food and in indoor environments. P. chrysogenum has been used industrially to produce penicillin. Recently, this author reported biological extracellular synthesis of copper oxide, silver and gold nanoparticles by using different Penicillium species and Enterobacteriacea family with fairly well-defined dimensions and good monodispersity (Honary et al., 2012a, 2012b, 2013a, 2013b). Biosynthesis of Metallic nanoparticles is very important due to their potential applications in catalysis, photonics, biomedicine and optics (Chauhan et al., 2011). Among all metallic nanoparticles, gold has a long history of use. Red colloidal gold has been used as medicine for revitalization in China and India (Bhattacharya and Murkherjee 2008). Gold nanoparticles, in particular, are of interest, mainly due to their stability under atmospheric conditions, resistance to oxidation and biocompatibility (Gericke and Pinches 2006). Furthermore, gold nanoparticles are used in diagnostic and drug delivery systems (Bhumkar et al., 2007). This study aimed to biosynthesize and optimizes the size of gold nanoparticles due to the unique size-dependent properties of gold nanoparticles that makes this material important in many areas of human activity (Fars et al., 2010). The average diameter of metal nanoparticles can be affected by some factors including $\mathrm{pH}$ of medium, salt concentration and temperature of shaker incubator in biological methods (Mohammadian et al., 2007; Rati et al., 2011). Response surface methodology (RSM) is a collection of mathematical and statistical techniques which has been known as a tool for modeling and determining the effects of factors and their interactions, on a certain response (e.g. Size) (Bezerraa et al., 2008). The mathematical methodology could propose the best condition for production of desirable metal nanoparticles. This method also could help us to reduce time and costs, and give more precise results. In this paper, RSM were applied to investigate the effect of concentration of $\mathrm{AuCl}_{4}, \mathrm{pH}$ of medium and temperature of shaker incubator on the average diameter size of gold nanoparticles. We believe that biosynthesis would absolutely be the first choice to form the nanoparticles for pharmaceutical applications if the methodology was optimized.

\section{Materials and Methods}

Yeast extract was purchased from Liofilchem, Italy. $\mathrm{AuCl}_{4}$ and other chemical reagents were purchased from Merck Germany. The pure colony of Penicillium crustosum was isolated from soil and approved by department of mycology and plant pathology of Sari Agriculture and Natural Resources University, Iran.

\section{Preparation and characterization of gold nanoparticles}

The fungus Penicillium crustosum was cultured on fluid czapex dox broth including $21 \mathrm{~g}$ sucrose and $3 \mathrm{~g}$ yeast extract in $1000 \mathrm{~mL}$ distilled water and incubated on a rotary shaker at $200 \mathrm{rpm}$ for ten days at $28{ }^{\circ} \mathrm{C}$. The culture was centrifuged at 10,000 rpm for $5 \mathrm{~min}$. Then the supernatant was separated from the mycelia to convert $\mathrm{AuCl}_{4}$ solution into nano-gold. $100 \mathrm{~mL}$ of different $\mathrm{AuCl}_{4}$ solution concentrations were added to $100 \mathrm{~mL}$ of supernatant according to the response surface methodology and incubated again for $24 \mathrm{~h}$ at $28^{\circ} \mathrm{C}$. In this step, the formed gold nanoparticles were centrifuged at 20,000 rpm by ultracentrifuge for $5 \mathrm{~min}$ to separate nano-golds from the solution. Then, the nanoparticles redispersed in double distilled water and centrifuged again. This action was carried out for three times to separate purified gold nanoparticles. Dynamic light scattering (DLS) was carried out to determine the size, polydispersity index (PDI), correlogram and intensity fluctuations of nanoparticles by a Zetasizer Nanoparticle Analyzer using Zetasizer 3600 at $25^{\circ} \mathrm{C}$ with a scattering angle of $90^{\circ}$ (Malvern instruments, UK). The surface and shape characterizations of gold nanoparticles were done using atomic force microscopy (AFM) under ambient conditions on a Veeco Innova, USA. The AFM characterization was carried out in a non-contact mode using silicon nitride tips with varying resonance frequencies at a linear scanning rate of $0.5 \mathrm{~Hz}$. Furthermore, the UV-Vis Spectrum measurements were carried out by an Ultraviolet (UV) spectroscopy (Genesys 2 spectrophotometer USA). Fouriertransform infrared (FT-IR) spectrum was obtained by mix- 
ing with potassium bromide at 1:100 ratios and compressed to a $2 \mathrm{~mm}$ semi-transparent disk for $2 \mathrm{~min}$. The FT-IR spectrum over the wavelength range $4000-400 \mathrm{~cm}^{-1}$ was recorded using an FT-IR spectrometer (Perkin Elmer, Germany).

\section{Experimental design and optimization}

The second-order designs such as central composite (CCD) and Box-Behnken (BBD) play an important role in response surface methodologies especially when the design economy and precise prediction variance are desired. BBD is a kind of rotatable design which uses just three levels of each factor. However, BBD unlike CCD can be expected to have poorer prediction ability in the corners of the cube that encloses the design. In the present study, Box-Behnken design was employed to study the effect of independent variables, i.e. $\mathrm{pH}$ of medium $\left(X_{1}\right)$, concentration of Au salt $\left(X_{2}\right)$ and temperature of incubator $\left(X_{3}\right)$ on the response (size of gold nanoparticles). A second order polynomial equation (Eq. (1)) was used to describe the effect of the above variables in linear, quadratic and cross product terms:

$Y=\beta_{0}+\sum_{i=1}^{k} \beta_{i} X_{i}+\sum_{i=1}^{k} \beta_{i j} X_{i}^{2}+\sum_{i<j}^{k} \sum_{j}^{k} \beta_{i j} X_{i} X_{j}+e$

where, $Y$ refers to average diameter size of nanoparticles, while $k$ the number of factors studied in the experiment, $b$ is regression coefficient and ' $e$ ' is residual error. When developing the regression equation, the test factors were coded according to the following equation (Eq. (2)):

$$
x_{i}=\frac{\left(X_{i}-X_{0}\right)}{\Delta X_{i}} i=1,2, \ldots, k
$$

where $x_{i}$ is the dimensionless value of an independent variable, $X i$ is the real value of an independent variable, $X_{0}$ is the real value of the independent variable at the center point, and $\Delta X i$ is the step change value (Gustavo, 1998; Lundstedt et al., 1998; Bezerraa et al., 2008). Box-behnken could not consider all the third order terms, because there are fully aliased with linear effects $\left(\right.$ e.g. $X_{1}^{3}=X_{1}$ ). However, when the analysis of second order terms are exhibiting lack of fit, to achieve a better fit, two effects of $X_{1}^{2} X_{3}$ and $X_{1} X_{2}^{2}$ entered into the model (Arshad et al., 2012). Regression coefficients were reviewed and approved using variance inflation factor (VIF) to avoid probable multicollinearity due to correlation of third order terms with linear effects. Table 1 showed three studied experimental variables at three levels. These variables were chosen as they were considered to have the most significant effect on the size of nanoparticles. The levels were selected based on knowledge that acquired from initial experimental trials. The statistical analysis was performed on the range scaled factor values of $[-1,+1]$. Design-Expert 7.0, trial version was used to analyze the experimental design data and calculation of predicted responses. The quality of the obtained
Table 1 - Variables and experimental design levels for response surface.

\begin{tabular}{lccc}
\hline Level & $\mathrm{X}_{1}{ }^{\mathrm{a}}$ & $\mathrm{X}_{2}$ & $\mathrm{X}_{3}$ \\
\hline-1 & 6 & 0.5 & 25 \\
0 & 7 & 1.75 & 31 \\
+1 & 8 & 3 & 37 \\
\hline
\end{tabular}

${ }^{\mathrm{a}} \mathrm{X}_{1}, \mathrm{X}_{2}$ and $\mathrm{X}_{3}$ refer to $\mathrm{pH}$ of medium, concentration of $\mathrm{AuCl}_{4}(\mathrm{mM})$ and temperature of shaker incubator $\left({ }^{\circ} \mathrm{C}\right)$, respectively.

polynomial model was expressed by the coefficient of determination $\left(\mathrm{R}^{2}\right)$, the value of adjusted- $\mathrm{R}^{2}$ of model, Fisher-ratio value $(\mathrm{F})$, and standard error of the estimate (SE). The significance of each term in the equation was to estimate the goodness of fit in each case. The analysis of variance tables was generated and the effect and regression coefficients of individual linear, quadratic and interaction terms were determined. The P-values of less than 0.05 were considered to be statistically significant. The regression coefficients were then used to make statistical calculations to generate contour and dimensional maps from the regression models.

\section{Results}

\section{Preparation and purification of gold nanoparticles}

The addition of Penicillium crustosum supernatant to $\mathrm{AuCl}_{4}$ solution led to the appearance of dark purple color after $24 \mathrm{~h}$ of reaction. The control sample (without gold ions) showed no change in color when incubated under same conditions. The UV-Vis spectrum exhibited an absorption band at around $527 \mathrm{~nm}$ (Figure 1A), which is a typical plasmon band for gold nanoparticles (Honary et al., 2013a). The UV-vis Spectrum of supernatant withought gold nanoparticles in low wavelength region, recorded from the reaction medium, exhibited an absorption band around $265 \mathrm{~nm}$ and it was attributed to aromatic amino acids of proteins (Figure 1B). The fluorescence spectrum of the synthesized gold nanoparticles showed a broad emission peak of $\mathrm{AuCl}_{4}$ nanoparticles at $551 \mathrm{~nm}$ when excited at $527 \mathrm{~nm}$ (Figure 1A).

The FT-IR spectrum of synthesized gold nanoparticles showed that nanoparticles manifests absorption peaks located at about 1407.36, 1629.91, 2922.26 and $3432 \mathrm{~cm}^{-1}$ in the region $450-4000 \mathrm{~cm}^{-1}$. The peaks at $3432 \mathrm{~cm}^{-1}$ assigned to $\mathrm{O}-\mathrm{H}$ stretching. The band at $2922.26 \mathrm{~cm}^{-1}$ associated with the $\mathrm{C}-\mathrm{H}$ stretch of the methylene groups of the protein. The band at 1407.36 and $1629.91 \mathrm{~cm}^{-1}$ corresponds to the N-H bend of primary amines due to carbonyl stretch in proteins (Figure 2).

The AFM micrographs of nanoparticles showed that the gold nanoparticles produced by Penicillium crustosum were spherical shaped with a mean average size under 100 nm (Figure 3) that was adapted with the result of DLS showed in Figure 4. 

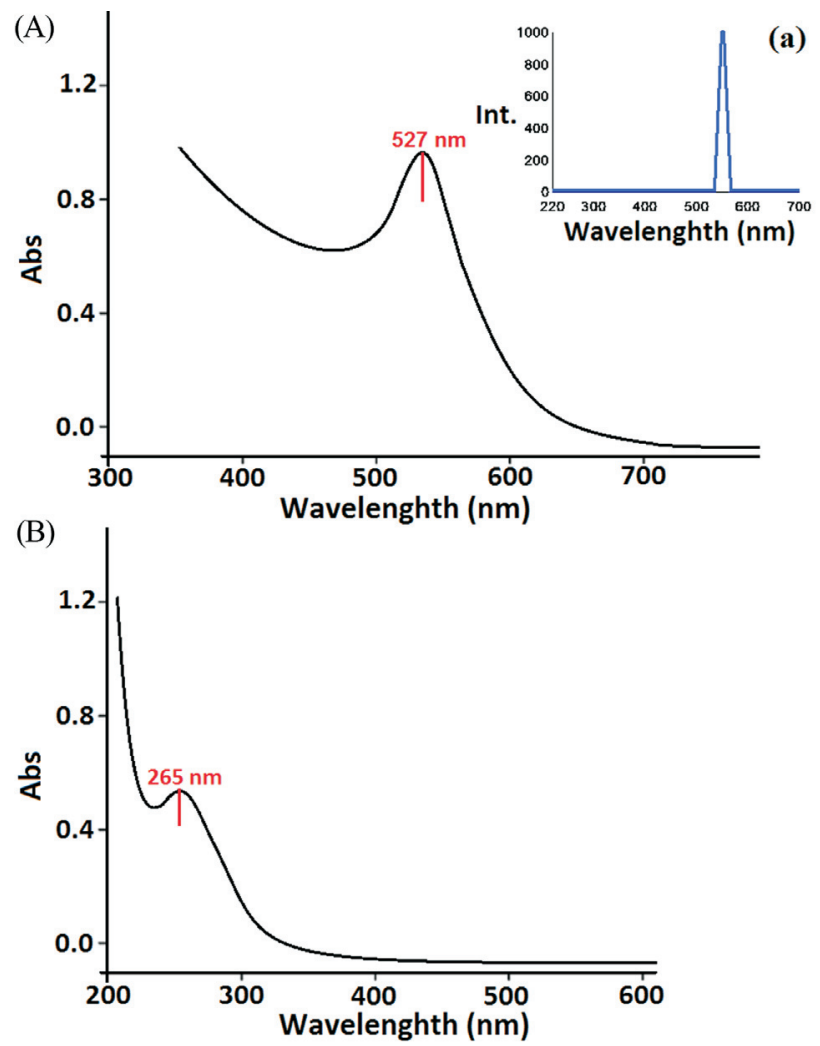

Figure 1 - A: UV-Visible absorption spectra of the synthesized gold nanoparticles. A(a): The fluorescence absorption spectrum of the synthesized gold nanoparticles. B: The culture medium of Penicillium crustosum.

Furthermore, dynamic light scattering (DLS) showed the fairly well-defined dimensions and good monodispersity of synthesized nano-golds. An example of DLS graphs was shown in Figure 4A with the polydispersity index (PDI) of 0.248. Besides, correlogram of nanoparticles which measured by zetasizer (Malvern instruments, UK), confirmed the monodispersity and small size of synthesized nano-golds (Figure 4A). The zeta potential of the gold nanoparticles was found at $-26.0 \mathrm{mV}$ (Figure 4B).

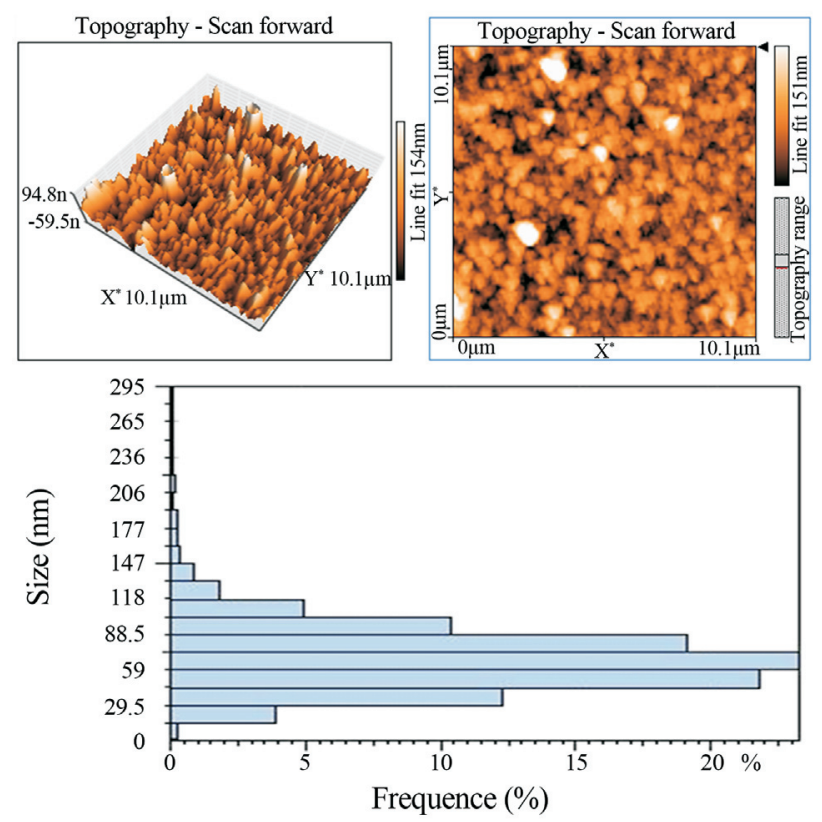

Figure 3 - The AFM image of gold nanoparticles in the solutions showed a mean average size under $100 \mathrm{~nm}$ that was adapted with the result of DLS showed in Figure 4.

\section{Optimization analysis}

The optimization of gold nanoparticles was performed by 15 experiments based on Box-Behnken design. The studied factors were the $\mathrm{pH}$ of medium, salt concentration $\left(\mathrm{AuCl}_{4}\right)$ and the temperature of shaker incubator. Table 2 showed the 15 experimental conditions and the average diameter of gold nanoparticles according to the experimental design. It was found out that trial 5 led to the minimum average size of gold nanoparticles among all.

The quadratic model was checked, using the Design-Expert 7.0, trial version for ANOVA and the results were shown in Table 3. The P-values were used as a tool to check the significance of each coefficient, which also indicated the interaction strength of each parameter. In the pres-

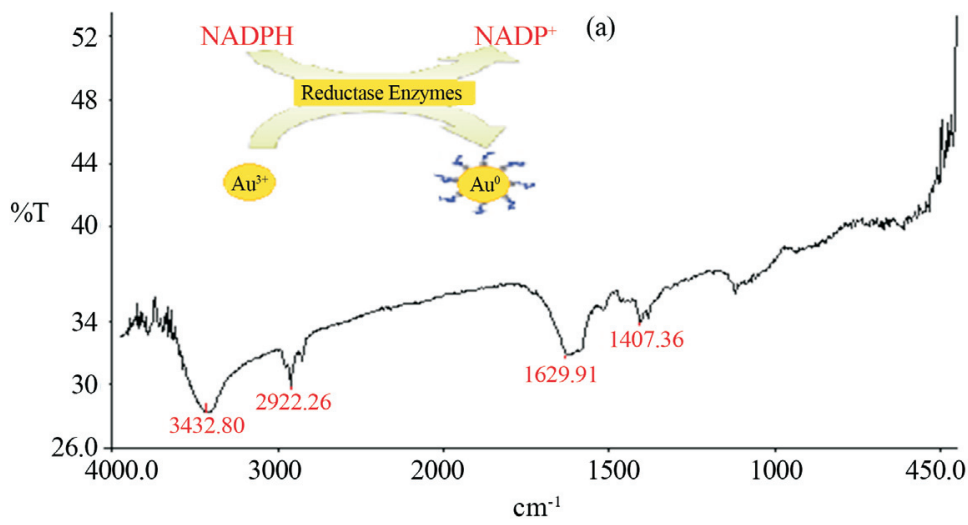

Figure 2 - FT-IR spectrum of gold nanoparticles synthesized by Penicillium crustosum. Figure 2(a): Schematic proposed mechanism of gold ions bioreduction via NADPH-dependent reductase enzymes. 
(4)
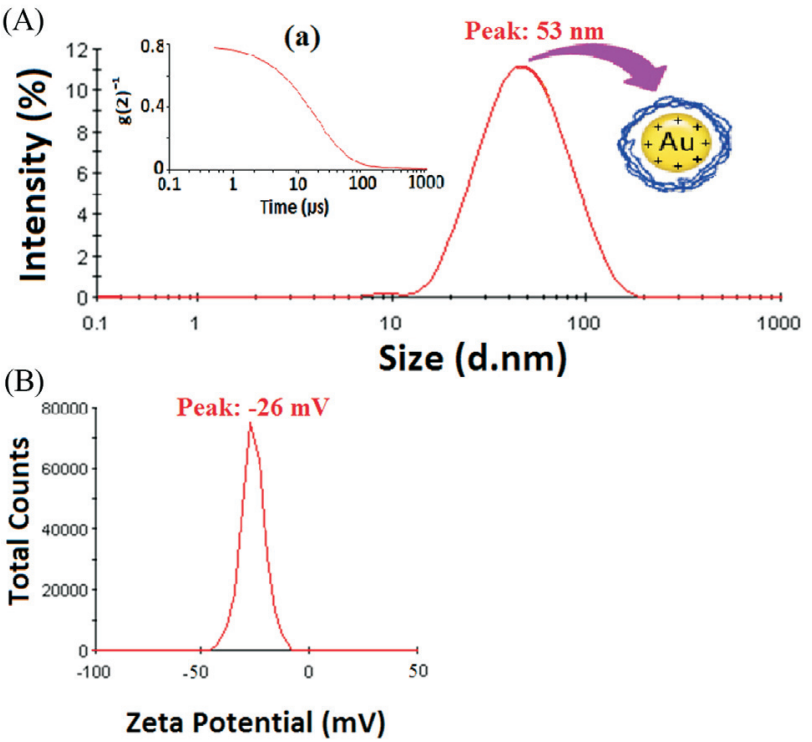

Figure 4 - A: Dynamic light scattering (DLS) of the gold nanoparticles. A(a): Correlogram of gold nanoparticles showed small synthesized particles in which the correlation of the signal decays more rapidly. B: The zeta potential of the gold nanoparticles.

ent study, the Fisher-ratio (2797.98) and P-values $(\mathrm{p}=0.0004)$ indicating statistical significance of the obtained model that demonstrated the relationship between the independent variables and the response, according to the regression model. Value of adjusted- $\mathrm{R}^{2}(0.9996) \mathrm{sug}$ gested that the total variation of $99.96 \%$ average diameter size of nanoparticles is attributed to the independent variables and only about $0.04 \%$ of the total variation cannot be explained by the model.

Table 2 - Experimental conditions for Box-Behenken design and average response for particle size.

\begin{tabular}{|c|c|c|c|c|}
\hline Trial & $X_{1}^{\mathrm{a}}$ & $X_{2}$ & $X_{3}$ & average diameter $(\mathrm{nm})$ \\
\hline 1 & + & + & 0 & 142 \\
\hline 2 & + & - & 0 & 169 \\
\hline 3 & - & & 0 & 203 \\
\hline 4 & - & - & 0 & 102 \\
\hline 5 & + & 0 & + & 53 \\
\hline 6 & + & 0 & - & 131 \\
\hline 7 & - & 0 & & 198 \\
\hline 8 & - & 0 & - & 149 \\
\hline 9 & 0 & & + & 203 \\
\hline 10 & 0 & & - & 211 \\
\hline 11 & 0 & - & + & 195 \\
\hline 12 & 0 & - & - & 117 \\
\hline 13 & 0 & 0 & 0 & 104 \\
\hline 14 & 0 & 0 & 0 & 103 \\
\hline 15 & 0 & 0 & 0 & 102 \\
\hline
\end{tabular}

${ }^{\mathrm{a}} X_{1}, X_{2}$ and $X_{3}$ refer to $\mathrm{pH}$ of medium, concentration of $\mathrm{AuCl}_{4}(\mathrm{mM})$ and temperature of shaker incubator $\left({ }^{\circ} \mathrm{C}\right)$, respectively.
Table 3 - Analysis of variance for the fitted quadratic polynomial model of optimization of size of biosynthesized gold nanoparticles.

\begin{tabular}{lccccc}
\hline Source & $\begin{array}{c}\text { Sum of } \\
\text { squares }\end{array}$ & $\begin{array}{c}\text { Degree of } \\
\text { freedom }\end{array}$ & $\begin{array}{c}\text { Mean } \\
\text { square }\end{array}$ & F-value & $\begin{array}{c}\text { Probability } \\
(\mathrm{p})>\mathrm{F}\end{array}$ \\
\hline Model & 33575.73 & 12 & 2797.98 & 2797.98 & 0.0004 \\
Pure error & 2.00 & 2 & 1.00 & & \\
$\mathrm{R}^{2}$ & 0.9999 & & & & \\
Adj R $^{2}$ & 0.9996 & & & & \\
\hline
\end{tabular}

The regression coefficients and the corresponding P-values were presented in Table 4. From the P-values, it could be concluded that all the independent variables studied $\left(X_{1}, X_{2}, X_{3}\right)$ and quadratic term $\left(X_{2}^{2}\right)$ significantly affected the average diameter of size. The analysis showed there were significant interactions between $X_{1}^{2} X_{2}, X_{1}^{2} X_{3}$ and $X_{1} X_{2}^{2}$. Based on the analysis of regression coefficients of the quadratic model depicted that $\mathrm{pH}$ of medium $\left(X_{1}\right)$ exhibited a negative relationship on the average diameter size, while concentration of $\mathrm{AuCl}_{4}(\mathrm{mM})\left(X_{2}\right)$ and temperature of shaker incubator $\left({ }^{\circ} \mathrm{C}\right)\left(X_{3}\right)$ exhibited a positive relationship on the average diameter size.

\section{Discussion}

\section{Preparation and Characterization of gold nanoparticles}

Formation of gold nanoparticles was apparent from the gradual changes in the color of incubated solution from pale yellow to dark purple, but the color of control remained practically unchanged during the entire incubation period. This color changes from pale yellow to dark purple

Table 4 - Regression coefficients and their significance of the quadratic model of optimization of size of biosynthesized gold nanoparticles.

\begin{tabular}{lcccc}
\hline Model term & $\begin{array}{c}\text { Coefficient } \\
\text { estimate }\end{array}$ & $\begin{array}{c}\text { Degree of } \\
\text { freedom }\end{array}$ & $\begin{array}{c}\text { Standard } \\
\text { error }\end{array}$ & $\begin{array}{c}\text { Probability } \\
(\mathrm{p}) \mathrm{F}\end{array}$ \\
\hline Intercept & +103.00 & 1 & 0.58 & 0.0004 \\
$X_{1}^{\mathrm{a}}$ & -40.75 & 1 & 0.50 & 0.0002 \\
$X_{2}$ & +25.50 & 1 & 0.50 & 0.0004 \\
$X_{3}$ & +17.50 & 1 & 0.50 & 0.0008 \\
$X_{1} X_{2}$ & -32.00 & 1 & 0.50 & 0.0002 \\
$X_{1} X_{3}$ & -31.75 & 1 & 0.50 & 0.0002 \\
$X_{2} X_{3}$ & -21.50 & 1 & 0.50 & 0.0005 \\
$X_{1}^{2}$ & +1.13 & 1 & 0.52 & 0.1632 \\
$X_{2}^{2}$ & +49.87 & 1 & 0.52 & 0.0001 \\
$X_{3}^{2}$ & +28.63 & 1 & 0.52 & 0.0003 \\
$X_{1}^{2} X_{2}$ & -7.00 & 1 & 0.71 & 0.0101 \\
$X_{1}^{2} X_{3}$ & -24.75 & 1 & 0.71 & 0.0008 \\
$X_{1} X_{2}^{2}$ & +42.25 & 1 & 0.71 & 0.0003 \\
\hline & & & &
\end{tabular}

${ }^{\mathrm{a}} X_{1}, X_{2}$ and $X_{3}$ refer to $\mathrm{pH}$ of medium, concentration of $\mathrm{AuCl}_{4}(\mathrm{mM})$ and temperature of shaker incubator $\left({ }^{\circ} \mathrm{C}\right)$, respectively. 
is attributed to surface plasmon resonance (SPR) (Moores and Goettmann, 2006; Honary et al., 2013a). With a specific energy, an electron will be pushed away from the metal, creating negative and positive regions (Figure 5).

The electron will naturally return to its original side, but during the process other electrons have also been moving. This phenomenon is defined as a particle: the surface plasmon (Pitark et al., 2005; Moores and Goettmann, 2006). The energy at which the electrons begin to oscillate is specific, and any wavelength of light at that energy will be absorbed. This absorption results a change in color far different than the original gold color of the base metal. In fact, SPR is an excitation of the localized surface plasmon in the metal surface resulting from shining a monochromatic light source onto the nanoparticles which is the result of the collective dipole oscillation of conduction electrons against the background of an ionic metal-core. The position of the absorption peak in the visible absorption spectrum of colloidal solutions can be related to the particle size and shape due to this resonance through SPR (Durán et al., 2005; Noguez, 2007; Mohanpuria et al., 2008). A commonly used characterization technique for gold colloid is ultraviolet-visible (UV-vis) spectroscopy, which excites the plasmon resonance responsible for the characteristic color of colloidal gold. It was found that the visible absorption spectrum of gold nanoparticles in this study had an absorption band at around $527 \mathrm{~nm}$ (Figure 1A). This absorption band, also known as the plasmon mode, gives to the colloidal gold its characteristic dark purple color. The location of this plasmon mode is highly dependent on the size, aggregation and shape of the gold nanoparticles (Durán et al., 2005; Noguez, 2007). The UV-vis spectrum of low wavelength region, recorded from the reaction medium, exhibited an absorption band around $265 \mathrm{~nm}$ and it was attributed to aromatic amino acids of proteins (Figure 1B). It is well known that the absorption band at $265 \mathrm{~nm}$ arises due to electronic excitation in tryptophan and tyrosine residue in the protein. This is in accordance with the results of other researchers (Ahmad et al., 2003; Honary et al., 2012a). This finding suggested indirect evidence to the ability of this secreted proteines and enzymes as a reductant to form gold nanoparticles. Furthermore, the fluorescence spectrum showed a broad emission peak of gold nanoparticles at $549 \mathrm{~nm}$ when excited at $527 \mathrm{~nm}$ (Figure 1A). The nature of

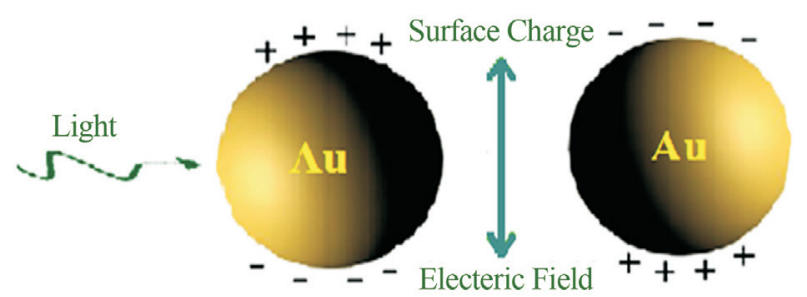

Figure 5 - Schematic representation of surface plasmon oscillation under the effect of an electromagnetic field. the emission band indicates that the proteins bound to the nanoparticle surface and those present in the solution exist in the native form (Durán et al., 2005). The FT-IR measurements were carried out to identify the possible bio-molecules responsible for capping and efficient stabilization of the metal nanoparticles synthesized by Penicillium crustosum. The spectrum represents different functional groups of adsorbed. It seems that the FT-IR spectrum reveal the presence of different functional groups like amid linkages and -COO- which may be between amino acid residues in protein and synthesized gold nanoparticles (Honary et al., 2013a). It is believed that the enzyme nitrate reductase could be responsible for bioreduction of metal ions and synthesis of nanoparticles (Ahmad et al., 2003; Honary et al., 2012a, 2012b, 2013a). The bioreduction of gold ions was found to be initiated by the electron transfer from the NADPH-dependent reductase as a electron carrier. Next, the gold ions $\left(\mathrm{Au}^{3+}\right)$ obtain electrons and are reduced to elemental gold $\left(\mathrm{Au}^{0}\right)$ nanoparticles (Figure 2A) (Tikariha et al., 2012). The secreted proteines and enzymes in the medium are responsibles not only for synthesis of metallic nanoparticles, but also for stabilization of nanoparticles against aggregation. Therefore, this green synthesis does not need any additive stabilizer which is a positive point in comparison with chemical methods (Ghorbani et al., 2011; Honary et al., 2011). The AFM micrographs of nanoparticles showed that the gold nanoparticles produced by Penicillium crustosum were spherical shaped and well distributed in solution (Figure 3). Figure 4A showed the DLS analysis of gold nanoparticles with an average diameter of $53 \mathrm{~nm}$ and PDI of 0.248 which demonstrated that the nanoparticles formed with fairly well-defined dimensions and good monodispersity. The zeta potential of the gold nanoparticles was found at $-26 \mathrm{mV}$ which provides the repulsive force as an electrostatic stabilization (Figure 4B). The correlogram of a measurement can give a lot of information about the sample. The time at which the correlation starts to significantly decay is an indication of the mean size of the sample. In small particles containing monodisperse samples, the correlation of the signal decays more rapidly (Figure 4A). But, the correlation of the signal in polydisperse samples takes a long time to decay.

\section{Optimization analysis}

Traditional optimization has been carried out by monitoring the effect of one factor at a time on an experimental response. While only one parameter is changed, others are kept at a constant level. This technique does not include the interactive effects among the variables studied and the complete influence of the parameter on the response could not be detected. Besides, the number of experiments necessary to conduct the research in this technique increases that lead to an increase in time and expenses (Mohammadian et al., 2007). The Box-Behnken is a suitable design for RSM since it allows estimation of the parameters of the quadratic 
model, building of sequential designs, detection of lack of fit of the model and use of blocks (Ferreira et al., 2007). A large Fisher-ratio value shows that most of the variation can be explained by a regression equation and a low $\mathrm{p}$-value $(<0.05)$ shows that the model is considered to be statistically significant (Zunlai et al., 2012). In the present study, the Fisher-ratio value of 2797.98 and value of $p=0.0004$ indicated statistical significance of a quadratic model. Therefore, this large F-value and low p-value demonstrated that the relationship between the independent variables and the response can be explained according to the regression model. In addition, Validation of optimized study performed using three confirmatory runs indicated very high degree of prognostic ability of mathematical methodology. Response surfaces were plotted using Design-Expert 7.0, trial version software to study the effects of parameters and their interactions on average diameter size of biosynthesized nanoparticles. The shapes of the contour plots provide a measurement for the significance of the mutual interactions between the variables. An elliptical contour plot indicates a significant interaction between variables. These three-dimensional plots and their respective contour plots provide a visual interpretation of the interaction between two variables and facilitate the location of optimum experimental conditions. The relationship between the experimental factors and response can be understood by examining the series of contour plots generated by holding temperature of shaker incubator $\left(\mathrm{X}_{3}\right)$ (Figure 6), concentration of $\mathrm{AuCl}_{4}\left(\mathrm{X}_{2}\right)$ (Figure 7$)$ and $\mathrm{pH}$ of medium $\left(\mathrm{X}_{1}\right)($ Figure 8$)$ at a constant level. The points on the corners and center of the Figures 6 to 8 represent experimental design points. The point with number 3 in the centre indicates that contour plots have been drawn when the value of the fixed

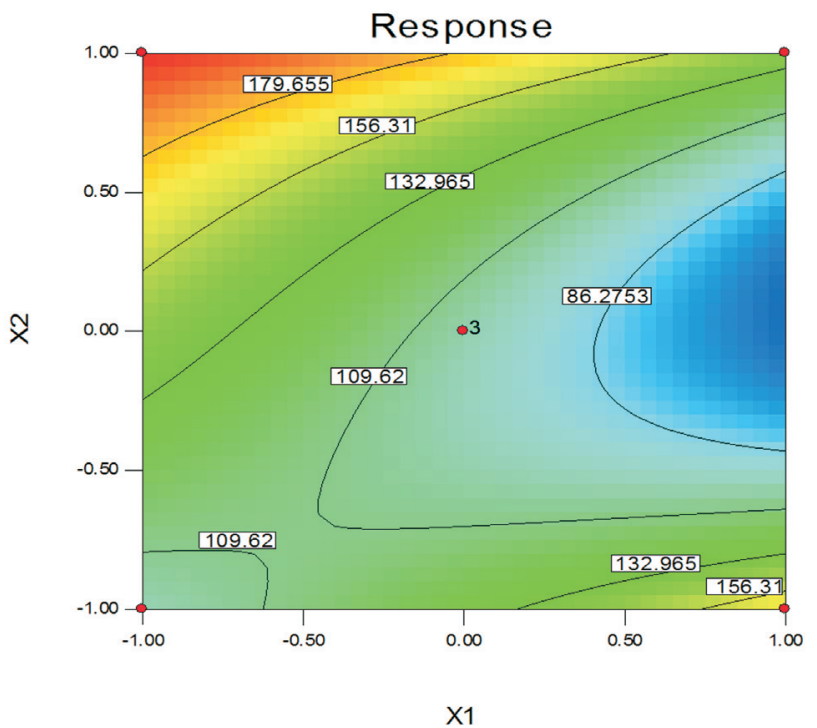

Figure 6 - Contour plot of $\mathrm{pH}$ of medium $\left(\mathrm{X}_{1}\right)$ and concentration of $\mathrm{AuCl}_{4}$ $(\mathrm{mM})\left(\mathrm{X}_{2}\right)$ on the size of gold nanoparticles, when temperature of shaker incubator $\left(\mathrm{X}_{3}\right)$ was maintained at $31^{\circ} \mathrm{C}$. variable is at the mid point of lowest and highest selected levels. From the Figures 6 and 7 show that as the $\mathrm{pH}$ of medium $\left(\mathrm{X}_{1}\right)$ increases, the average diameter size decreases, but at the higher $\mathrm{pH}$ values, the size slightly increases. This may be due to less activity of reducer enzymes at low and high temperature. Similarly, as shown in Figures 6 and 8, when concentration of $\mathrm{AuCl}_{4}(\mathrm{mM})\left(\mathrm{X}_{2}\right)$ increases, the average diameter size decreases, but begin to increase at higher concentration. This might be explained that increasing of salt concentration allows particle growth at a faster rate. Moreover, Particles in higher salt concentration maybe aggregate and produce bigger particles. From the

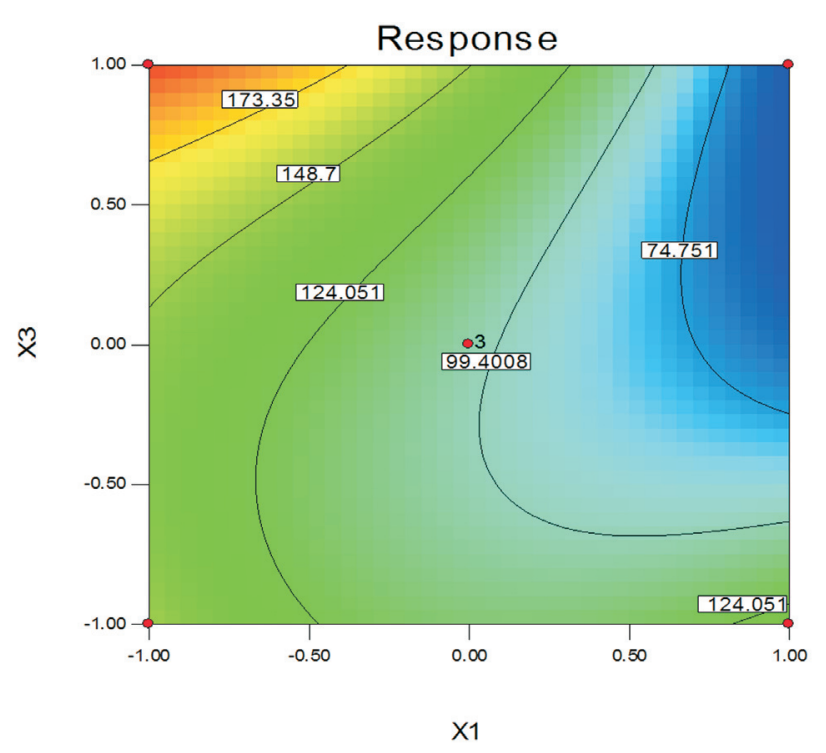

Figure 7 - Contour plot of $\mathrm{pH}$ of medium $\left(\mathrm{X}_{1}\right)$ and temperature of shaker incubator $\left({ }^{\circ} \mathrm{C}\right)\left(\mathrm{X}_{3}\right)$ on the size of gold nanoparticles, when concentration of $\mathrm{AuCl}_{4}\left(\mathrm{X}_{2}\right)$ was maintained at $1.75 \mathrm{mM}$.

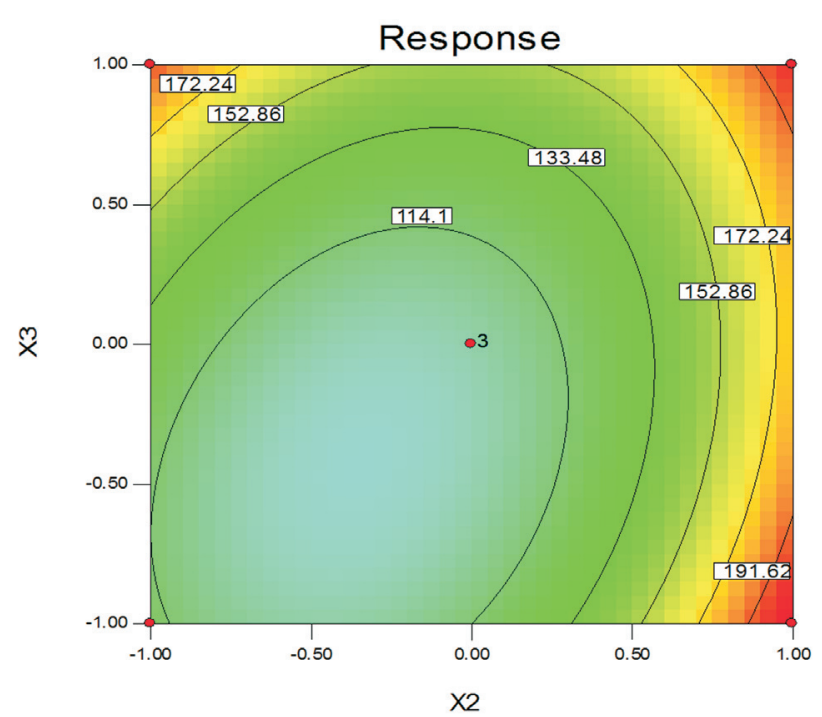

Figure 8 - Contour plot of concentration of $\mathrm{AuCl}_{4}(\mathrm{mM})\left(\mathrm{X}_{2}\right)$ and temperature of shaker incubator $\left({ }^{\circ} \mathrm{C}\right)\left(\mathrm{X}_{3}\right)$ on the size of gold nanoparticles, when $\mathrm{pH}$ of medium $\left(\mathrm{X}_{1}\right)$ was maintained at value of 7 . 
practical point of view, it would be better if the metal ions could be prepared in lower concentration, leading to the formation of smaller nanometer-sized particles in the solution (Honary et al., 2012a). Figure 8 shows an elliptical contour plot represents that interaction between concentration of $\mathrm{AuCl}_{4}(\mathrm{mM})\left(\mathrm{X}_{2}\right)$ and temperature of shaker incubator $\left({ }^{\circ} \mathrm{C}\right)\left(\mathrm{X}_{3}\right)$ has a significant effect on the average diameter size. A circular contour plot represents that the interaction between the corresponding variables is negligible while elliptical contour plot indicates significant interactions (Muralidhar et al., 2001). Elliptical contour plots obtained from the data of the present study clearly shows that the mutual interactions between the variables are significant. Therefore, it could be concluded that the empirical model is adequate to describe average size of synthesized nanoparticles developed by response surface.

\section{Conclusion}

Compared to other methods, 'green' method seems to be less costly, simpler and yet more functional, and also would require less energy and fewer raw materials for production. We have described here a green method for the synthesis of gold nanoparticles exploiting Penicillium crustosum. The results show that interaction between $\mathrm{pH}$ $\left(X_{1}\right)$, concentration of $\mathrm{AuCl}_{4}\left(X_{2}\right)$ and temperature of shaker incubator $\left(X_{3}\right)$ all have significant effects on the average size of gold nanoparticles. Besides, it was shown that statistically designed experiments involves several factors simultaneously are more efficient when studying two or more factors. This could be of major importance in industry, where experiments could be very expensive and time consuming. Hence, the mathematical methodology could be quite efficient and useful for the optimization of preparation of gold nanoparticles and provide efficient parameters for scaled-up industrial production of metallic nanoparticles. Furthermore, the extracellular synthesis of nanoparticles could be highly valuable from the viewpoint of large-scale operations and easy downstream processing though still in need of further optimization and characterization for full understanding of their whole potential.

\section{Acknowledgments}

This work was financially supported by Traditional Medicine \& Materia Medica Research Center (TMRC) Shaheed Beheshti University of Medical Sciences, Tehran, Iran.

\section{References}

Ahmad A, Mukherjee P, Senapati S, Mandal D, Khan MI, Kumar R, Sastry M (2003) Extracellular biosynthesis of silver nanoparticles using the fungus Fusarium oxysporum. Colloids Surf B Biointerf 28:313-318.
Arshad HM, Akhtar M, Gilmour SG (2012) Augmented boxbehnken designs for fitting third-order response surfaces. Commun. Stat. Theory. 41:4225-4239.

Bezerraa MA, Santelli RE, Oliveiraa EP, Villar LS, Escaleiraa LA (2008) Response surface methodology (RSM) as a tool for optimization in analytical chemistry. Talanta 76:965-977.

Bhattacharya R, Murkherjee P (2008) Biological properties of "naked" metal nanoparticles. Adv Drug Deliv Rev 60:1289-1306.

Bhumkar DR, Joshi HM, Sastry M, Pokharkar VB (2007) Chitosan reduced gold nanoparticles as novel carriers for transmucosal delivery of insulin. Pharm Res 24:1415-1426.

Chauhan A, Zubair S, Tufail S, Sherwani A, Sajid M, Raman SC, Azam A, Owais M (2011) Fungus-mediated biological synthesis of gold nanoparticles: potential in detection of liver cancer. Int J Nanomedicine 6:2305-2319.

Rai M, Kovics G (2010) Progress in mycology. Scientific publishers, India.

Durán N, Marcato PD, Alves OL, Souza GI, Esposito E (2005) Mechanistic aspects of biosynthesis of silver nanoparticles by several Fusarium oxysporum strains. J. Nanobiotechnology 3:8.

Fars KA, Awwad A, Radwan IAA (2010) Biopharmaceutical applications of nanogold. Saudi Pharm. J. 18:179-193.

Ferreira SLC, Bruns RE, Ferreira HS, Matos GD, David JM, Brand GC, Silva EGP da, Portugal LA, Reis PS dos, Souza AS, Santos WNL dos (2007) Box-Behnken design: An alternative for the optimization of analytical methods. Analytica Chimica Acta 597:179-186.

Gade AX, Bonde P, Ingle AP, Marcato PD, Duran N, Rai MK (2008) Exploitation of Aspergillus niger for synthesis of silver nanoparticles. J Biobased Mater Bio 2:243-247.

Gericke M, Pinches A (2006) Microbial production of gold nanoparticles. Gold Bull 39:22-28.

Ghorbani HR, Safekordi AA, Attar H, Rezayat Sorkhabadi SM (2011) Biological and non-biological methods for silver nanoparticles synthesis. Chem. Biochem. Eng. Q. 25:317326.

Gustavo GA (1998) Two level factorial experimental designs based on multiple linear regression models: a tutorial digest illustrated by case studies. Analytica Chimica Acta 360:227-241.

Honary S, Barabadi H, Gharaei-Fathabad E, Naghibi F (2012a) Green synthesis of copper oxide nanoparticles using Penicillium aurantiogriseum, Penicillium citrinum and Penicillium waksmanii. Dig J Nanomater Bios 7:999-1005.

Honary S, Gharaei-Fathabad E, Khorshidi Paji Z, Eslamifar M (2012b) A novel biological synthesis of gold nanoparticle by Enterobacteriaceae family. Trop J Pharm Res 11:887-891.

Honary S, Gharaei-Fathabad E, Barabadi H (2013a) Fungusmediated synthesis of gold nanoparticles: a novel biological approach to nanoparticle synthesis. J Nanosci Nanotechnol 13:1427-1430.

Honary S, Barabadi H, Gharaei-Fathabad E, Naghibi F (2013b) Green synthesis of silver nanoparticles induced by the fungus Penicillium citrinum. Trop j pharm Res 12:7-11.

Honary S, Ghajar K, Khazaeli P, Shalchian P (2011) Preparation, characterization and antibacterial properties of silver-chitosan nanocomposites using different molecular weight grades of chitosan. Trop J Pharm Res 10:69-74. 
Ingle A, Gade A, Bawaskar M, Rai M (2009) Fusarium solani: A novel biological agent for the extracellular synthesis of silver nanoparticles. J Nanopart Res 11:2079-2085.

Lundstedt T, Seifert E, Abramo L, Thelin B, Nyström A, Pettersen J, Bergman R (1998) Experimental design and optimization. Chemom. Intell. Lab. Syst. 42:3-40.

Mohammadian A, Shojaosadati SA, Habibi Rezaee M (2007) Fusarium oxysporum mediates photogeneration of silver nanoparticles. Scientia Iranica 14:323-3266.

Mohanpuria P, Rana NK, Yadav SK (2008) Biosynthesis of nanoparticles: technological concepts and future applications. J Nanopart Res 10:507-517.

Moores A, Goettmann F (2006) The plasmon band in noble metal nanoparticles: an introduction to theory and applications. New J Chem 30:1121-1132.

Muralidhar RV, Chirumamila RR, Marchant R, Nigam P (2001) A response surface approach for the comparison of lipase production by Candida cylindracea using two different carbon sources. Biochem. Eng. J. 9:17-23.

Noguez C (2007) Surface plasmons on metal nanoparticles: the influence of shape and physical environment. J. Phys. Chem 111:3806-3819.

Pitark JM, Silkin VM, Chulkov EV, Echenique PM (2005) Surface plasmons in metallic structures. J Optic Pure Appl Optic 7:73-84.

Priyadarshini S, Gopinath V, Meera Priyadharsshini N, Mubarak Ali D, Velusamy P (2013) Synthesis of anisotropic silver nanoparticles using novel strain, Bacillus flexus and its biomedical application. Colloids Surf B Biointerf 102:232-237.

Rati RN, Nilotpala P, Debadhyan B, Kshyama MP, Srabani M, Lala BS, Barada KM (2011) Green synthesis of silver nanoparticle by Penicillium purpurogenum NPMF: the process and optimization. J Nanopart Res 13:3129-3137.
Sagar G, Ashok B (2012) Green synthesis of silver nanoparticles using Aspergillus niger and its efficacy against human pathogens. Eur J Exp Biol 2:1654-1658.

Schuster E, Dunn-Coleman N, Frisvad JC, Van Dijck PW (2002) On the safety of Aspergillus niger- $a$ review. Appl Microbiol Biotechnol 59:426-435.

Seifert K (1996) Fusarium interactive key. Agriculture Agrifood Canada pp 1-30.

Tikariha S, Singh S, Banerjee S, Vidyarthi AS (2012) Biosynthesis of gold nanoparticles, scope and application: a review. IJPSR 3:1603-1615.

Vigneshwaran N, Ashtaputre NM, Varadarajan PV, Nachane RP, Paralikar KM, Balasubramanya RH (2007) Biological synthesis of silver nanoparticles using the fungus Aspergillus flavus. Mater Lett 61:1413-418.

Vladimír F, Lucia R, Juraj L (2012) Response surface methodology as optimization tool in study of competitive effect of $\mathrm{Ca}^{2+}$ and $\mathrm{Mg}^{2+}$ ions in sorption process of $\mathrm{Co}^{2+}$ by dried activated slud. JMBFS 1:1235-1249.

Zhao Y, Jiang Y, Fang Y (2006) Spectroscopy property of Ag nanoparticles. Acta A 65:1003-1006.

Zhang X, Yan S, Tyagi RD, Surampalli RY (2011) Synthesis of nanoparticles by microorganisms and their application in enhancing microbiological reaction rates. Chemosphere 82:489-494.

Zunlai S, Jichang L, Yanbing L (2012) Optimization of ultrasonic-assisted extraction of phillyrin from Forsythia suspensa using response surface methodology. J Med Plants Res 6:1633-1644.

All the content of the journal, except where otherwise noted, is licensed under a Creative Commons License CC BY-NC. 